\title{
OBSERVATION OF TeV GAMMA RAYS FROM THE CRAB NEBULA WITH MILAGRO USING A NEW BACKGROUND REJECTION TECHNIQUE
}

\author{
R. Atkins, ${ }^{1}$ W. Benbow, ${ }^{2,3}$ D. Berley, ${ }^{4}$ E. Blaufuss,${ }^{4}$ J. Bussons,${ }^{4,5}$ D. G. Coyne,${ }^{2}$ R. S. Delay, ${ }^{6}$ T. DeYoung, ${ }^{2}$ \\ B. L. Dingus, ${ }^{7}$ D. E. Dorfan, ${ }^{2}$ R. W. Ellsworth,${ }^{8}$ A. Falcone,,${ }^{, 10}$ L. Fleysher,${ }^{11}$ R. Fleysher,${ }^{11}$ G. Gisler,${ }^{7}$ \\ M. M. Gonzalez, ${ }^{1}$ J. A. Goodman, ${ }^{4}$ T. J. Haines,${ }^{7}$ E. Hays,${ }^{4}$ C. M. Hoffman, ${ }^{7}$ L. A. Kelley, ${ }^{2}$ R. W. Laird, ${ }^{1}$ \\ J. McCullough, ${ }^{2,12}$ J. E. McEnery, ${ }^{1,13}$ R. S. Miller, ${ }^{9}$ A. I. Mincer, ${ }^{11}$ M. F. Morales, ${ }^{2,14}$ \\ P. Nemethy, ${ }^{11}$ D. Noyes,${ }^{4}$ J. M. Ryan,${ }^{9}$ F. W. Samuelson,${ }^{7}$ M. Schneider,${ }^{2}$ B. Shen,${ }^{15}$ \\ A. Shoup,${ }^{6}$ G. Sinnis,${ }^{7,16}$ A. J. Smith,${ }^{4}$ G. W. Sullivan, ${ }^{4}$ O. T. Tumer, ${ }^{15}$ K. Wang, ${ }^{15,17}$ \\ M. Wascko, ${ }^{15,18}$ D. A. Williams, ${ }^{2}$ S. Westerhoff, ${ }^{2,19}$ M. E. Wilson, ${ }^{1}$ \\ $\mathrm{X} . \mathrm{Xu},{ }^{7}$ AND G. B. $\mathrm{YODH}^{6}$ \\ Received 2003 May 2; accepted 2003 June 11
}

\begin{abstract}
The recent advances in $\mathrm{TeV}$ gamma-ray astronomy are largely the result of the ability to differentiate between extensive air showers generated by gamma rays and hadronic cosmic rays. Air Cerenkov telescopes have developed and perfected the "imaging" technique over the past several decades. However, until now no background rejection method has been successfully used in an air shower array to detect a source of TeV gamma rays. We report on a method to differentiate hadronic air showers from electromagnetic air showers in the Milagro gamma-ray observatory, based on the ability to detect the energetic particles in an extensive air shower. The technique is used to detect $\mathrm{TeV}$ emission from the Crab Nebula. The flux from the Crab Nebula is estimated to be $2.68\left( \pm 0.42^{\text {stat }} \pm 1.4\right.$ sys $) \times 10^{-7}(E / 1 \mathrm{TeV})^{-2.59} \mathrm{~m}^{-2} \mathrm{~s}^{-1} \mathrm{TeV}^{-1}$, where the spectral index is assumed to be as given by the HEGRA collaboration.
\end{abstract}

Subject headings: gamma rays: observations — instrumentation: detectors -

ISM: individual (Crab Nebula) — stars: neutron — supernova remnants

\section{INTRODUCTION}

Ground-based gamma-ray astronomy has its roots in the pioneering work of the 1950s (Galbraith \& Jelley 1953; Nesterova \& Chudakov 1955). However, it was not until the late 1980s that the first convincing detection of a source of $\mathrm{TeV}$ gamma rays was made with a ground-based instrument. The innovation that led to this discovery was the development of the "imaging" technique in atmospheric Cerenkov telescopes (Hillas 1985; Weekes \& Turver 1977). This technique can distinguish air showers induced by gamma rays from those induced by hadrons (protons and

1 University of Wisconsin, Madison, WI 53706.

2 University of California, Santa Cruz, CA 95064.

${ }^{3}$ Current address: Max Planck Institute, Heidelberg, Germany.

${ }^{4}$ University of Maryland, College Park, MD 20742.

5 Current address: Université de Montpellier II, Montpellier, France.

${ }^{6}$ University of California, Irvine, CA 92717.

${ }^{7}$ Los Alamos National Laboratory, Los Alamos, NM 87545.

8 George Mason University, Fairfax, VA 22030.

${ }^{9}$ University of New Hampshire, Durham, NH 03824-3525.

${ }^{10}$ Current address: Purdue University, West Lafayette, IN 47907.

${ }^{11}$ New York University, New York, NY 10003.

${ }_{12}$ Current address: Cabrillo College, Aptos, CA 95003.

${ }^{13}$ Current address: Goddard Space Flight Center, Greenbelt, MD 20771.

${ }^{14}$ Current address: Massachusetts Institute of Technology, Cambridge, MA 02139.

15 University of California, Riverside, CA 92521.

${ }^{16}$ Corresponding author (Los Alamos National Laboratory, MS 803, Los Alamos, NM 87545; gus@lanl.gov).

${ }^{17}$ Current address: Stanford Linear Accelerator Center, Menlo Park, CA 94025

${ }^{18}$ Current address: Louisiana State University, Baton Rouge, LA 70803 .

${ }^{19}$ Current address: Columbia University, New York, NY 10027. heavier nuclei). The imaging technique categorizes air showers by the shape and orientation of the Cerenkov light pool as observed in the image plane of an optical telescope (an air Cerenkov telescope). This technique was used by the Whipple experiment to detect $\mathrm{TeV}$ gamma-ray emission from the Crab Nebula (Weekes et al. 1989). Since the initial discovery of the Crab Nebula, at least five other sources of $\mathrm{TeV}$ gamma rays have been detected (Hoffman et al. 1999; Ong 1998).

Despite the success of imaging air Cerenkov telescopes, they have several limitations. Because they are optical instruments, they can observe the sky only on clear, dark (moonless) nights (the typical duty cycle of these instruments is between $5 \%$ and $10 \%$ ), and they can observe only a small fraction of the sky at any one time (of order $4 \times 10^{-3}$ sr). In contrast, an instrument that detects the air shower particles that reach the ground, known as an extensive air shower (EAS) array, can operate 24 hours a day and can simultaneously view the entire overhead sky. Past efforts to distinguish hadronic and gamma-ray-induced air showers in EAS arrays have relied on the identification of muons. The CASA and CYGNUS arrays used shielded detectors to identify muons present in hadronic air showers at energies above $100 \mathrm{TeV}$ (Alexandreas et al. 1992; Borione et al. 1994). While the CASA array achieved very high levels of background rejection (rejecting $94 \%$ of the cosmic-ray background above $115 \mathrm{TeV}$ and $99.93 \%$ of the background above $1175 \mathrm{TeV}$, while retaining over $72 \%$ of the gammaray showers), no signals were observed in its data (Borione et al. 1997). It is generally believed that the absence of observed sources at these high energies is due to the absorption of high-energy photons by the low-energy background radiation fields ( $3 \mathrm{~K}$ and infrared) and/or the steeply falling spectra of astrophysical sources. 
The Milagro detector is an EAS array that is sensitive to much lower energy primary photons $(>100 \mathrm{GeV}$, with a median energy near $4 \mathrm{TeV}$ ) and can therefore detect more distant sources (up to redshift $\sim 0.3$ ) and sources that have intrinsic upper limits to the energy of the gamma rays produced. Here we report on the development of a technique to identify and reject hadronic background events in Milagro. We demonstrate the efficacy of the technique with a detection of $\mathrm{TeV}$ emission from the Crab Nebula.

\section{THE MILAGRO DETECTOR}

The Milagro gamma-ray observatory has 723 photomultiplier tubes (PMTs) submerged in a 24 million liter water reservoir. The detector is located at the Fenton Hill site of Los Alamos National Laboratory, about 35 miles west of Los Alamos, New Mexico, at an altitude of $2630 \mathrm{~m}$ above sea level $\left(750 \mathrm{~g} \mathrm{~cm}^{-2}\right)$. The reservoir measures $80 \mathrm{~m} \times$ $60 \mathrm{~m} \times 8 \mathrm{~m}$ (depth) and is covered by a light-tight barrier. Each PMT is secured by a Kevlar string to a grid of sandfilled PVC sitting on the bottom of the reservoir. The PMTs are arranged in two layers, each on a $2.8 \mathrm{~m} \times 2.8 \mathrm{~m}$ grid. The top layer of 450 PMTs (under $1.4 \mathrm{~m}$ of water) is used primarily to reconstruct the direction of the air shower. By measuring the relative arrival time of the air shower across the array, the direction of the primary cosmic ray can be reconstructed with an accuracy of roughly 0.75 . The bottom layer of 273 PMTs (under $6 \mathrm{~m}$ of water) is used primarily to discriminate between gamma-ray-initiated air showers and hadronic air showers. For a more detailed description of Milagro, see Atkins et al. (2001).

An EAS at ground level is composed primarily of electrons, positrons, and low-energy gamma rays. The gamma rays outnumber the electrons and positrons in the air shower by a factor of $\sim 4$. Since a radiation length in water is $\sim 36 \mathrm{~cm}$, the bulk of the gamma rays convert to electrons and positrons before reaching the top layer of PMTs. The relativistic charged particles emit Cerenkov radiation in a $41^{\circ}$ cone. The top layer of the array detects the Cerenkov radiation from the charged particles in the air shower with high efficiency ( $\sim 50 \%$ of all electromagnetic particles that enter the pond are detected). The combination of high particle detection efficiency and a moderately high altitude gives Milagro a lower energy threshold than other air shower arrays. Milagro is sensitive to gamma rays with energies above $100 \mathrm{GeV}$. With a nominal trigger threshold of 60 PMTs (of the 450 in the top layer), the event rate is $1700 \mathrm{~Hz}$.

The amount of light detected at a PMT depends on the detailed trajectories of the particles in a region around that PMT. Since electromagnetic particles may penetrate close to the PMTs in the top layer of the pond, the fluctuations in the amount of detected light are large. Thus, the top layer is not very useful for imaging the energy flow in an EAS. In contrast, only muons, showering hadronic particles, and energetic electromagnetic particles (such as those near the core of an air shower) can penetrate to the bottom layer of the pond. Thus, the bottom layer can be used to image the energy deposited by the air shower. The image of the EAS in the bottom layer of Milagro is a good discriminator against hadronic cosmic rays. The method described below, based on the "compactness" of the EAS in the bottom layer, eliminates the bulk of the events initiated by the hadronic cosmic rays $(\sim 90 \%)$, while retaining $\sim 50 \%$ of the events initiated by gamma rays.

\section{IDENTIFICATION AND REJECTION OF HADRONIC EVENTS}

When a hadronic cosmic ray enters the atmosphere, it interacts with the nuclei in the air. These interactions lead to the production of charged pions that can decay into muons and neutrinos. In addition, multi-GeV hadronic particles may also reach the ground. In contrast, when a gamma ray enters the atmosphere, the interactions with the nuclei in the air are almost purely electromagnetic, resulting in an air shower that contains mostly lower energy electrons, positrons, and gamma rays.

Muons have great penetrating power; muons with energy above $1.2 \mathrm{GeV}$ (at ground level) reach the bottom layer of PMTs. The muons that reach the bottom layer illuminate a relatively small number of neighboring PMTs. Hadrons shower in the detector, also yielding large pulses in a small group of neighboring PMTs in the bottom layer. Monte Carlo simulations indicate that $80 \%$ of cosmic-ray-induced air showers and $6 \%$ of gamma-ray-induced air showers that trigger Milagro have at least one muon or hadron entering the pond.

Figure 1 shows six illustrative Monte Carlo events imaged in the bottom layer of Milagro. The simulations were generated over a zenith angle range of $0^{\circ}-45^{\circ}$. (Throughout this paper an event selection is made on the Monte Carlo events identical to that made on the data: the number of struck PMTs in the top layer must be greater than 60 , the number of PMTs used in the angular fit must be greater than 20, and for gamma rays, the events must be reconstructed within 1.2 of their true direction.) CORSIKA 6.003 (Knapp \& Heck $1993)^{20}$ is used to generate the extensive air shower, and GEANT 3.21 is used to track the interactions of the particles through the water and to simulate the response of the detector. The simulated cosmic-ray events are comprised of protons and helium nuclei: roughly $20 \%$ of the event triggers are due to helium nuclei. The top three events are gammaray-induced events, and the bottom three are protoninduced events. The area of each square is proportional to the number of photoelectrons (PEs) registered in the corresponding PMT, and the area is saturated at 300 PEs. The events were selected so that the proton events have properties (core location and number of PMTs struck in the top layer) similar to the gamma-ray events directly above them. Table 1 gives the relevant parameters for each event. The final parameter $C$ (for compactness) is defined in the next paragraph.

It can be seen that the gamma-ray events have relatively smooth PE distributions in the bottom layer and the hadronic events have well-defined clumps of high-intensity regions. Since there is no optical barrier between the two layers of PMTs, light generated in the top layer can be registered in the bottom-layer PMTs. To minimize the impact of this optical cross talk between the top and bottom layers of PMTs, a PE threshold is applied to the PMTs in the bottom layer. Four different threshold values have been investigated: 1, 2, 3, and 4 PE thresholds have been applied to the

\footnotetext{
${ }^{20}$ For an up-to-date version of the user's manual, see http://www-ik3.fzk.de/ heck/corsika.
} 

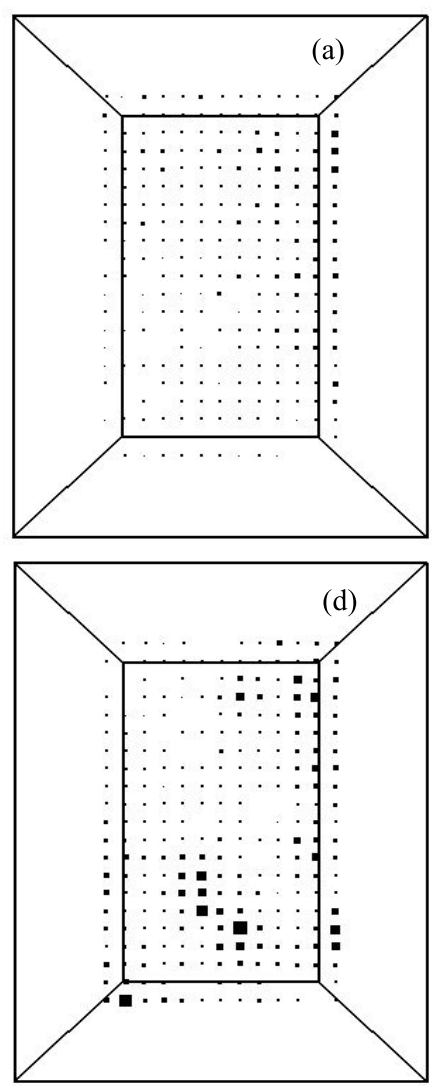
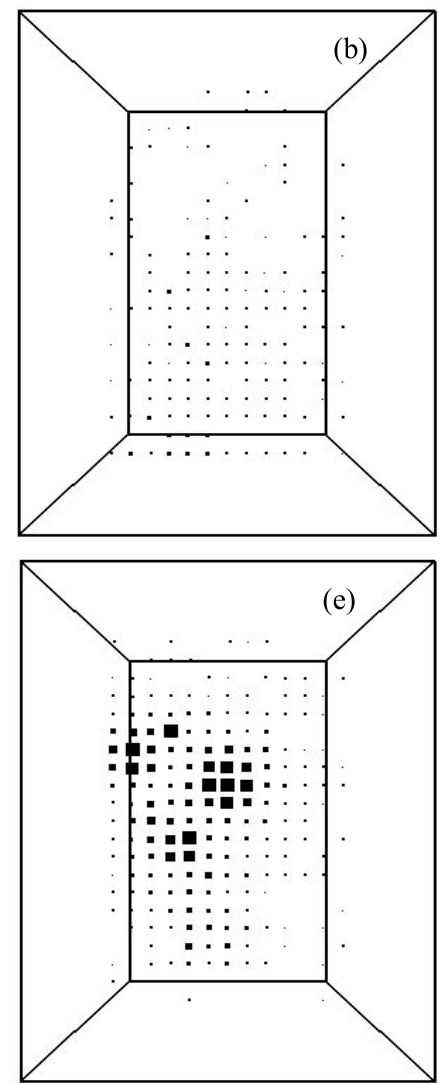
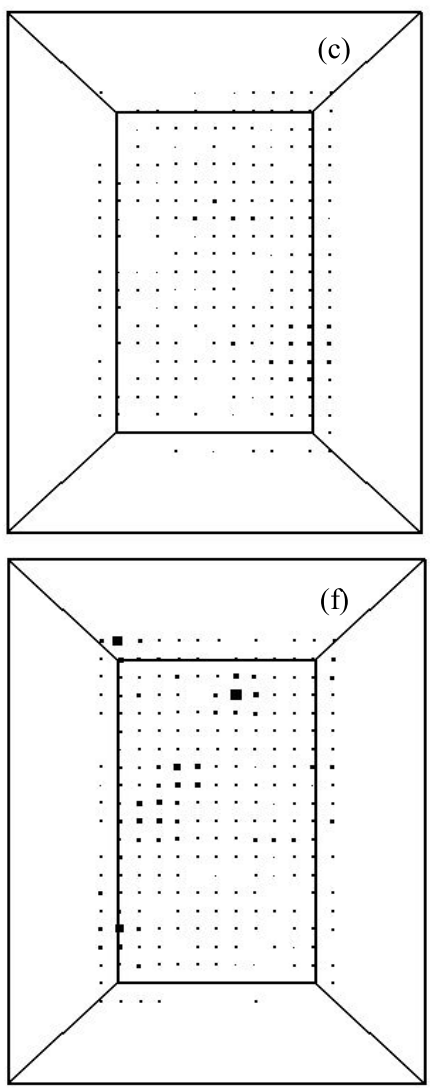

FIG. 1.-Gamma-ray (top row) and proton (bottom row) events imaged in the bottom layer of Milagro. The area of each square is proportional to the pulse height registered in each PMT. The proton and gamma-ray events have been selected to have similar properties in the top layer of the detector. See the text and Table 1 for details.

parameter $C=\mathrm{NB} / \mathrm{PEMax}_{B}$. The term NB is the number of PMTs in the bottom layer with a pulse height above the $\mathrm{PE}$ threshold, and $\mathrm{PEMax}_{B}$ is the number of PEs in the PMT with the maximum number of PEs in the bottom layer. This parameter should yield a small value for events where a few PMTs in the bottom layer are struck with a large amount of light and a high value for events where many PMTs are struck with a small amount of light. For each threshold one can determine a $C$-value below which events are characterized as hadron-like and rejected. The value of such a cut is determined by calculating the ratio of the significance of a signal after the cut is applied to the significance of the signal before the cut is applied to the data. This

TABLE 1

Properties of Events Shown in Figure 1

\begin{tabular}{|c|c|c|c|c|}
\hline Panel & $\begin{array}{l}\text { Energy } \\
(\mathrm{TeV})\end{array}$ & Core Position & $N_{\text {Top }}$ & $C$ \\
\hline 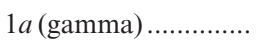 & 1.84 & $1.5 \mathrm{~m}$ from pond & 326 & 3.2 \\
\hline 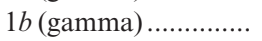 & 0.89 & In pond & 115 & 4.55 \\
\hline 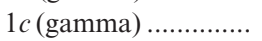 & 17.0 & $60 \mathrm{~m}$ from pond & 292 & 6.0 \\
\hline $1 d$ (proton) $\ldots \ldots \ldots \ldots \ldots$ & 36.0 & $4 \mathrm{~m}$ from pond & 366 & 0.36 \\
\hline $1 e$ (proton) ................... & 0.13 & In pond & 180 & 0.03 \\
\hline 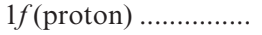 & 14.0 & $55 \mathrm{~m}$ from pond & 302 & 0.96 \\
\hline
\end{tabular}

Note.-The events were selected to have similar core locations and a similar number of PMTs struck in the top layer. ratio is commonly referred to as the quality factor $(Q)$ of the cut. For large numbers of events, $Q=\epsilon_{\gamma} /\left(\epsilon_{p}\right)^{1 / 2}$, where $\epsilon_{\gamma}$ is the fraction of gamma-ray events retained and $\epsilon_{p}$ is the fraction of hadron-induced events retained.

Because the quality factor is poor for a PE threshold of 1 and the fraction of gamma-ray events retained is $20 \%$ or less for PE thresholds of 3 and 4, a PE threshold of 2 is used for the data analysis and in the remainder of this paper. The resulting parameter $C$ is referred to as compactness in the remainder of the paper and in Table 1.

Figure 2 shows the compactness distribution for gammaray events and cosmic-ray events (from Monte Carlo simulations), along with the observed distribution from the data. There is reasonable agreement between the Monte Carlo cosmic-ray events and the data. The $C$-distribution for the data has slightly fewer events at larger values of $C$ than the simulations predict, and a larger fraction of the Monte Carlo events have $C$-values near zero. The precise shape of the $C$-distribution for the data is sensitive to the absolute value assigned to $\operatorname{PEMax}_{B}$ and therefore to the absolute calibration of the detector. Such an absolute calibration is difficult, and in practice it is observed that the $C$-distribution of the data changes slightly with detector calibrations, detailed trigger conditions, and the number of nonworking PMTs. These variations have an insignificant impact on the overall quality factor of the cut, which is sensitive only to the fraction of events reconstructed with a $C$-value of 2.5 (see below). Over the observed detector variations, this fraction changes by at most $2 \%$. 


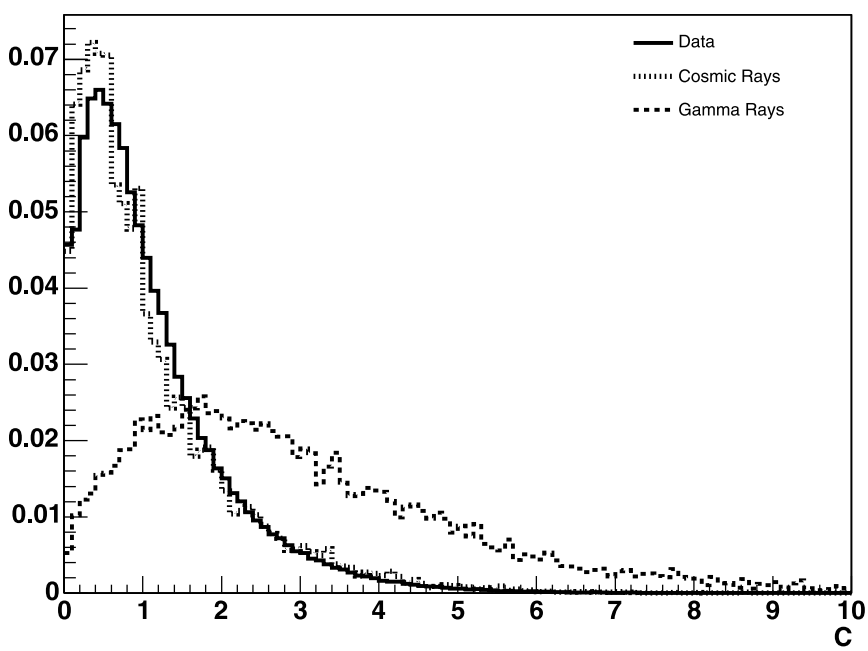

FIG. 2.-The $C$-distributions for Monte Carlo gamma-ray showers, Monte Carlo cosmic-ray showers, and data. All of the histograms have been normalized to have unit area.

Figure 3 shows the efficiencies for retaining data, cosmic rays, and gamma rays as a function of $C$, and Figure 4 shows the $Q$-value as a function of the minimum value of $C$ required to retain an event. In each of these distributions there is generally good agreement between the Monte Carlo cosmic-ray events and the data. Requiring events to have $C>2.5$ rejects $90 \%$ of the simulated cosmic-ray-induced air showers that trigger Milagro and $91.5 \%$ of the data (for this data sample), and retains $51 \%$ of the gamma-rayinduced air showers. This results in a predicted $Q$-value of 1.6 comparing Monte Carlo cosmic-ray events to Monte Carlo gamma-ray events, and 1.7 comparing the data to Monte Carlo gamma-ray events.

\section{PROPERTIES OF COMPACTNESS}

The efficiency of the compactness parameter is a function of the energy of the primary gamma ray. At low gamma-ray energies, only a few PMTs in the bottom layer have more than 2 PEs, so events with a low value of PEMax ${ }_{B}$ may have $C<2.5$. In addition, at lower energies the only gamma rays

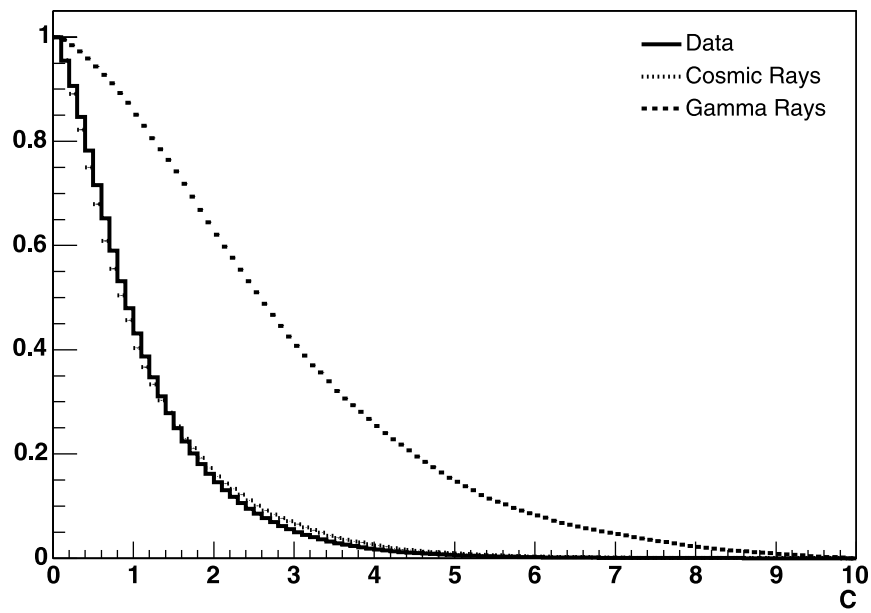

Fig. 3.-Fraction of Monte Carlo gamma rays, Monte Carlo cosmic rays, and data with $C$-values greater than $x$-axis value.

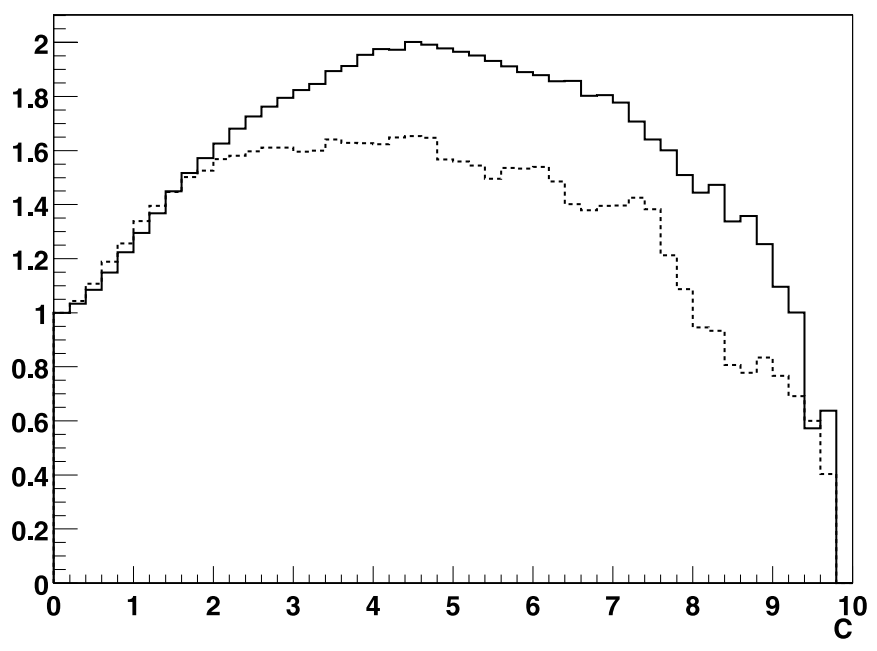

Fig. 4.-Quality factor $Q$ as a function of $C$. Events with $C$ greater than $x$-axis value are retained. The dashed line compares Monte Carlo gamma rays to Monte Carlo cosmic rays, and the solid line compares Monte Carlo gamma rays to data.

that trigger the detector tend to have their cores on the pond. The core of an electromagnetic shower typically contains the most energetic particles in the air shower. These particles can penetrate close to the bottom layer and again lead to events with $C<2.5$. In contrast, it is found that the $C$-distribution for cosmic-ray-induced events is relatively independent of energy. Figure 5 shows the efficiency of the compactness cut as a function of primary energy for gamma-ray-and cosmic-ray-initiated events. One sees that the cut does not reach $50 \%$ efficiency until $\sim 4 \mathrm{TeV}$, close to the median energy for gamma-ray showers. The efficiency for cosmic-ray-initiated events is nearly independent of energy. The same figure shows the triggered energy distribution of gamma rays generated on an $E^{-2.4}$ spectrum that are reconstructed within 1.2 of their true direction.

The compactness is also a function of the position of the core of the air shower. In particular, events with cores that

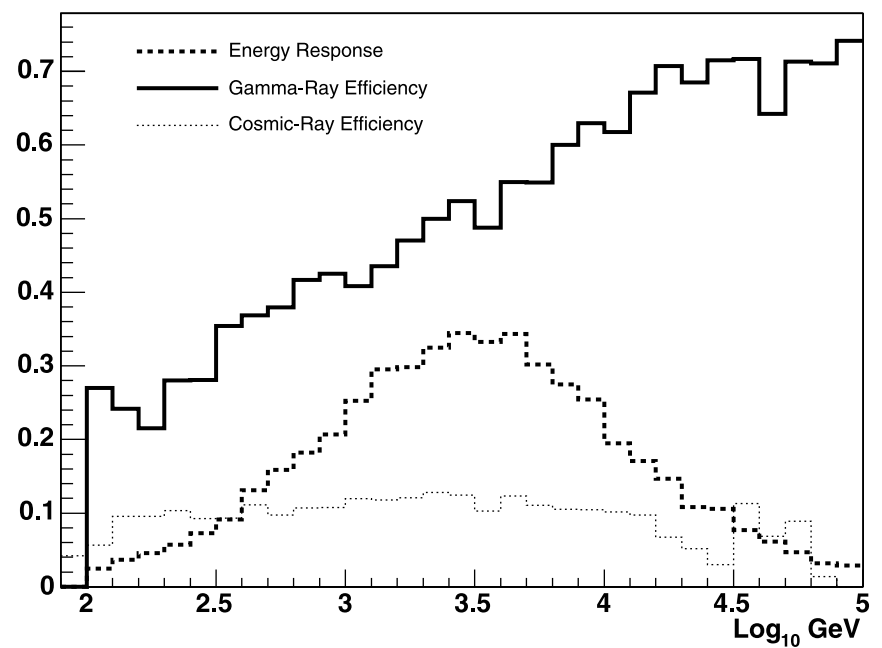

FIG. 5.-Fraction of gamma rays and cosmic rays retained by the compactness cut $(C>2.5)$ as a function of primary energy. The dashed line (labeled "Energy Response") shows the energy distribution of the gamma rays that trigger Milagro. The gamma rays were generated with an $E^{-2.4}$ spectrum. 

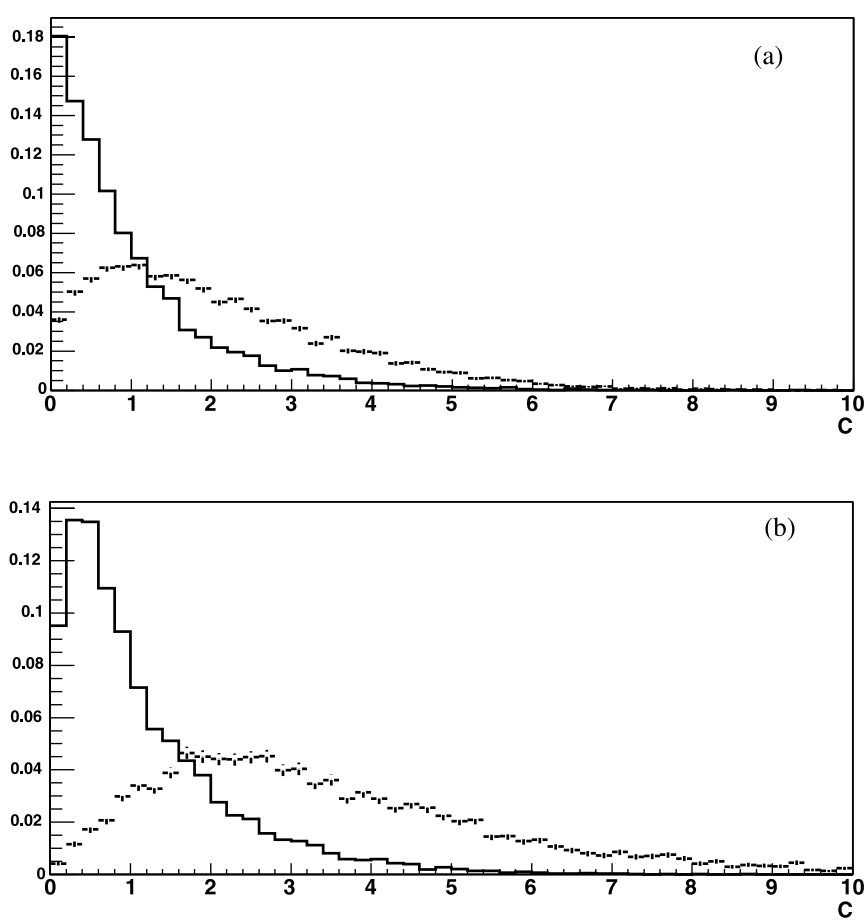

FIG. 6.-Compactness distributions of protons (solid line) and gamma rays (dotted line). (a) Events with their cores in the pond. (b) Events with their cores off the pond.

land on the pond tend to have smaller values of compactness (i.e., more hadron-like) than events with cores off the pond. As a result, the quality factor for events with cores on the pond is substantially smaller than for events with cores off the pond. Figure 6 shows the compactness distributions for protons and gamma rays (from Monte Carlo) for events with cores on the pond (top) and events with cores off the pond (bottom), and Figure 7 shows the quality factor as a function of the cut imposed on the compactness parameter (all events with compactness less than the $x$-axis value are rejected) for events with cores on and off the pond. The quality factor for events with cores off the pond is $\sim 50 \%$ higher than for the events with cores on the pond. If one

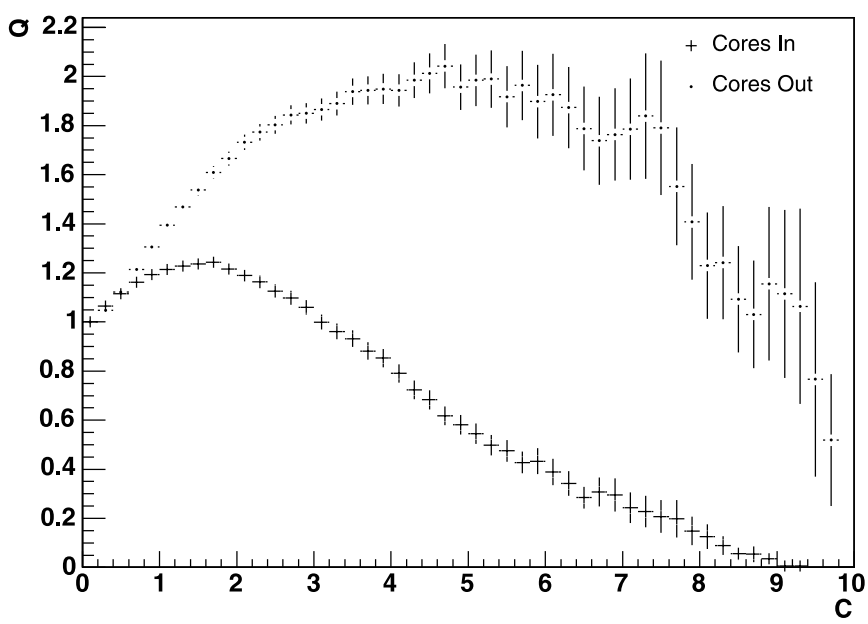

FIG. 7.-The $Q$-factor for events with their cores on and off the pond rejects all events with $C<2.5,35 \%$ of gamma-ray events with cores on the pond survive while $63 \%$ of gamma-ray events with cores off the pond survive. Similarly, for cosmic rays, $9 \%$ of events with cores on the pond survive and $12 \%$ of events with cores off the pond survive. At present, the detector is too small to reliably locate the cores of air showers, although an array of water tanks that surrounds the pond is currently under construction. This array will contain the bulk of the air showers that trigger Milagro and will be able to reliably locate the core of the EAS that triggers Milagro.

Finally, the dependence of compactness on the zenith angle of the air shower has been investigated. Since the energy threshold of the instrument is a function of zenith angle, and the number of muons generated in a hadronic cascade rises with primary energy, one might expect the rejection to improve with larger zenith angles. Figure 8 shows the compactness distributions for four different ranges of zenith angles. Figure $8 a$ shows the compactness for zenith angles less than $15^{\circ}$, Figure $8 b$ for events with zenith angles between $15^{\circ}$ and $25^{\circ}$, Figure $8 c$ for events with zenith angles between $25^{\circ}$ and $35^{\circ}$, and Figure $8 d$ for events with zenith angles between $35^{\circ}$ and $45^{\circ}$. The distributions look relatively similar. The quality factor as a function of the compactness cut is shown in Figures $9 a-9 d$ for the four zenith angle ranges. The three smaller zenith angle ranges give very similar quality factors; only the bin at the largest zenith angles $\left(35^{\circ}-45^{\circ}\right)$ gives a significantly different quality factor. However, at the compactness value selected for background rejection $(C>2.5)$, all four zenith angle ranges give a similar $Q$. Table 2 gives the efficiencies (and quality factors) of each of the zenith angle ranges for cosmic-ray and gamma-ray events for $C>2.5$.

\section{APPLICATION TO THE CRAB NEBULA}

The background rejection method derived above is applied to a search for $\mathrm{TeV}$ gamma rays from the $\mathrm{Crab}$ Nebula. While several different values for the flux have been reported, all observations to date indicate that the flux from the Crab Nebula is constant. This feature makes the Crab Nebula a useful source for calibrating the sensitivity of different instruments.

The data set begins on 1999 June 18 and ends on 2002 September 13. Because of detector down time, the effective exposure during this time interval is 917 days. The hardware trigger in Milagro requires 60 PMTs to fire within $200 \mathrm{~ns}$. The arrival time and pulse height are recorded for each hit PMT. The angular fit is an iterative one in which PMTs that make a large contribution to the $\chi^{2}$ of the fit are removed and the event is refitted. The number of PMTs remaining in the fit is called $n_{\text {fit }}$. The angular resolution of the detector is dependent on $n_{\text {fit }}$. (For details on the fitting procedure, see Atkins et al. 2000.) For this analysis, we reject events with $n_{\text {fit }}<20$, retaining roughly $91 \%$ of the data. From studies of the space angle difference in the reconstructed direction from independent, interleaved (like a checkerboard) portions of the detector, the shape of the Moon shadow, and Monte Carlo simulations, we estimate the angular resolution of the instrument to be 0.75 for all events with $n_{\text {fit }}>20$. For square bins the optimal bin is 2.8 times this wide (Alexandreas et al. 1993), or 2.1 in declination $(\delta)$ by $2.1 / \cos (\delta)$ in right ascension (R.A.). Since the angular response of the detector is not Gaussian, only $50 \%$ of events 

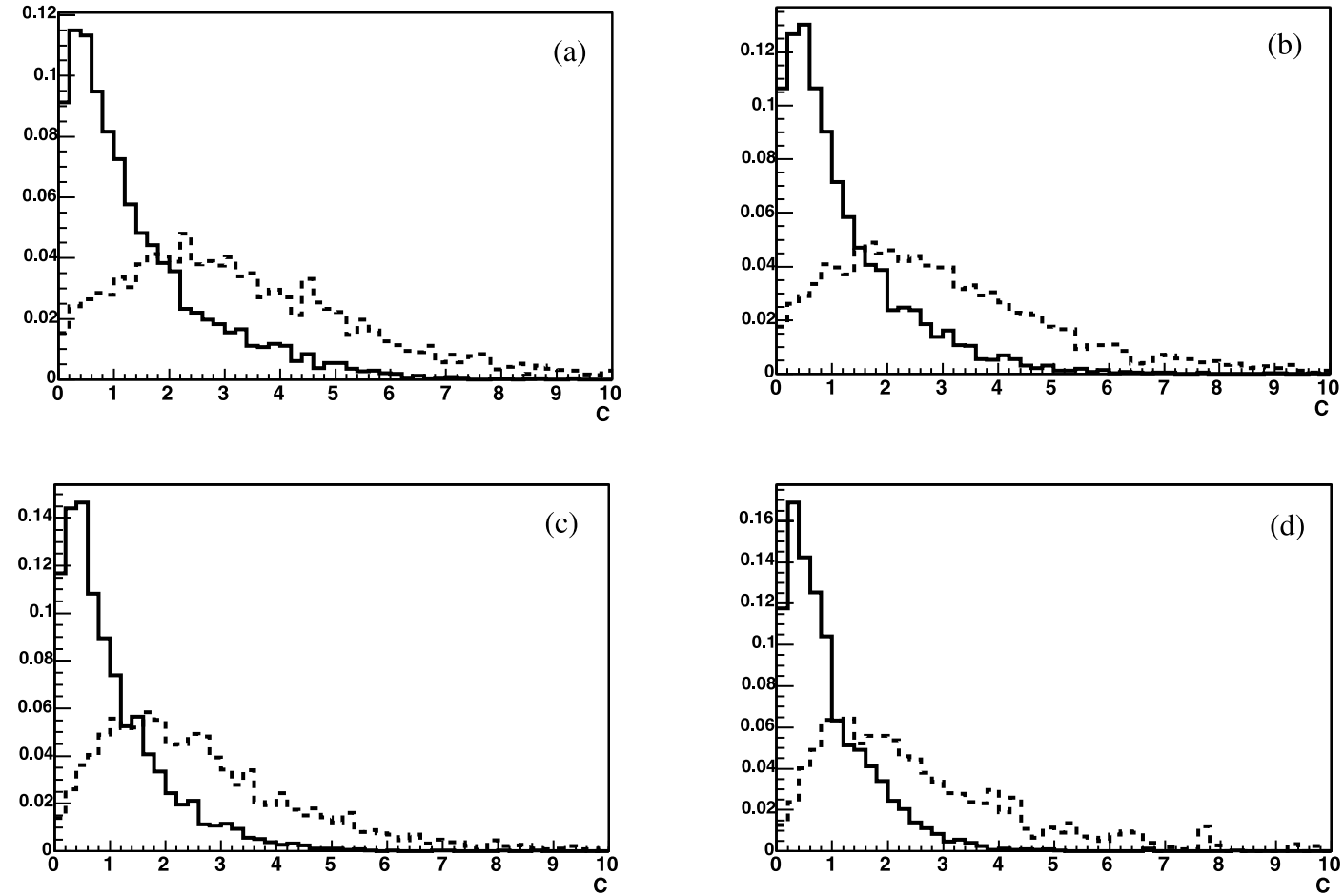

FIG. 8.-Compactness distributions for four zenith angle ranges of cosmic-ray (solid line) and gamma-ray (dotted line) showers. (a) Zenith angles less than $15^{\circ}$. (b) Zenith angles between $15^{\circ}$ and $25^{\circ}$. (c) Zenith angles between $25^{\circ}$ and $35^{\circ}$. (d) Zenith angles between $35^{\circ}$ and $45^{\circ}$.

from a source are reconstructed within this bin (for a detector with a Gaussian response, $72 \%$ of the events from the source would be reconstructed within this bin).

The analysis proceeds by counting the number of events that fall within the right ascension and declination bands defined by the bin centered on the Crab Nebula. This number is then compared with an estimate of the background. To estimate the background, a method known as "direct integration" is used. The event rate in the detector is a function of the local coordinates (hour angle [ha] and declination) and time. The technique assumes that the acceptance of the detector (expressed in local coordinates) is independent of the trigger rate over a $2 \mathrm{hr}$ period and that the cosmic

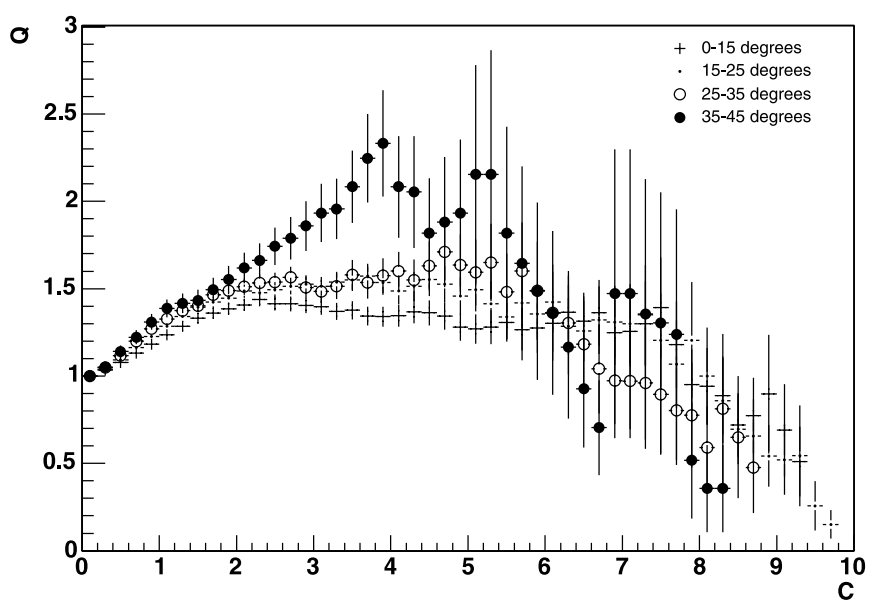

FIG. 9.-Quality factor as a function of the compactness cut for four different zenith angle ranges. rays form an isotropic background. Then one can write

$$
N_{\exp }[\text { R.A., } \delta]=\iint E(\text { ha, } \delta) R(t) \epsilon(\text { ha, R.A., } t) d t d \Omega,
$$

where $E($ ha, $\delta)$ is the efficiency or acceptance of the detector and is simply the probability that an event comes from the differential angular element $d \Omega=d($ ha $) d(\delta)$. The term $R(t)$ is the event rate of the detector as a function of time, and $\epsilon$ (ha, R.A., $t)$ is 1 if the hour angle, right ascension, and sidereal time are such that the event falls within the right ascension, declination bin of interest, and zero otherwise. The function $E($ ha, $\delta)$ is found by binning the local sky in $0.1 \times 0.1$ bins and counting (and normalizing) the number of events that fall within each bin (and pass any cuts made on the data) over the $2 \mathrm{hr}$ integration period. The integral is evaluated by summing the efficiency map (after the appropriate rotation is applied to convert hour angle to right ascension) over the observed events that pass the cuts imposed on the data. The error in the estimate of the background arises from the error in the determination of the detector acceptance, $E($ ha, $\delta)$. The significance of the excess

TABLE 2

EFFiciencies For COSMIC-RAY AND GAMma-RAy Events For the Cut $C>2.5$ For the Four Ranges of Zenith ANgles

\begin{tabular}{|c|c|c|c|}
\hline $\begin{array}{l}\text { Zenith Angle Range } \\
\text { (deg) }\end{array}$ & $\begin{array}{c}\text { Gamma-Ray } \\
\text { Efficiency } \\
(\%)\end{array}$ & $\begin{array}{c}\text { Proton } \\
\text { Efficiency } \\
(\%)\end{array}$ & $\begin{array}{l}\text { Quality } \\
\text { Factor }\end{array}$ \\
\hline $0-15 \ldots$ & 61 & 18 & 1.44 \\
\hline 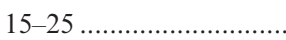 & 55 & 13 & 1.53 \\
\hline $25-35 \ldots \ldots \ldots \ldots \ldots \ldots \ldots \ldots \ldots$ & 47 & 9 & 1.57 \\
\hline 35-45 ……………..... & 42 & 6 & 1.71 \\
\hline
\end{tabular}


TABLE 3

Results of the Analysis of Data from the Crab Nebula

\begin{tabular}{crrcc}
\hline \hline Data Selection & On Source & Background & Excess & $\begin{array}{c}\text { Significance } \\
(\sigma)\end{array}$ \\
\hline All data .................. & $18,374,036$ & $18,365,694$ & 8342 & 1.9 \\
$C>2.5 \ldots \ldots \ldots \ldots \ldots \ldots . . .$. & $2,119,449$ & $2,109,732$ & 9717 & 6.4 \\
\hline
\end{tabular}

NoTE.-The results are given for all data and for data with $C>2.5$.

is calculated using the prescription of $\mathrm{Li} \& \mathrm{Ma}$ (1983), where the factor $\alpha$, the ratio of time on source to time spent off source, is $1 / 11.21$ and we have excluded the region around the Crab Nebula from our estimation of the background. A period of $2 \mathrm{hr}$ for the background was chosen to obtain sufficient statistics for the background estimation while minimizing the systematic errors arising from changes in the acceptance of the detector over the integration period.

The results of the Crab Nebula analysis are given in Table 3. The results are given both before and after the compactness cut has been applied to the data. Figure 10 shows a map of the region around the Crab Nebula after the compactness cut has been applied. The gray scale corresponds to the number of standard deviations in excess (or deficit) from each region of the sky. At each point in the figure the excess (or deficit) is integrated over a square bin of size 2.1 in declination and $2.1 / \cos (\delta)$ in right ascension. While the $C$-cut removed $88.5 \%$ of the data, in rough agreement with the Monte Carlo simulations, the efficiency for gamma rays appears to be greater than 1 . This nonphysical result arises from the large fluctuations in the background level (the low significance of the observed excess in the data before the application of the compactness cut). These same background fluctuations also explain the apparently large (and unphysical) quality factor of 3.3. (Since the efficiency for background events to pass the compactness cut is well determined from the data, $11.5 \%$, and the gamma-ray efficiency is bounded by 0 and 1 , the quality factor must be bounded by 0 and 2.9.) The low statistical significance of the excess in the uncut data precludes one from establishing firm limits on the gamma-ray efficiency of the compactness cut, and all that can be said is that the measurement is marginally consistent with the Monte Carlo prediction. The efficiency of the compactness parameter for gamma rays has also been investigated by examining the excess from the Crab Nebula as a function of compactness. For $C$-values above 1 , there is good agreement between the data and the Monte Carlo simulations.

The observed excess can be used to estimate the flux of $\mathrm{TeV}$ gamma rays from the Crab Nebula. The observed excess includes a small contamination from the source in the background estimation. This is a result of the finite angular resolution of the detector and the method used to estimate the background. Monte Carlo studies show that $16 \%$ of the signal events fall outside of the source bin but within the declination strip used to estimate the background. Therefore, the background level should be lowered (or the excess should be increased) by 138 events $(0.16 \times 9717 \alpha)$. Since the energy resolution of Milagro is relatively poor, it is not meaningful to fit the shape of the spectrum. Instead, four different functions have been assumed for the spectral shape, and the differential flux coefficient is determined for each of these spectral shapes. The four spectral functions are $d N / d E \propto E^{-2.49}$ and $d N / d E \propto E^{-2.44-0.151 \log _{10} E}$ from the Whipple collaboration (Hillas et al. 1998), $d N / d E \propto$ $E^{-2.59}$ from the HEGRA collaboration (Aharonian et al. 2000), and $d N / d E \propto E^{-2.62}$ from the Tibet collaboration (Amenomori et al. 1999). Since the response of Milagro is dependent on zenith angle, Monte Carlo simulations are used to estimate the effective area of Milagro as a function of energy, averaged over a transit of the Crab. For a source with spectrum of $f(E)$, the double integral over energy and time

$$
I_{0} \iint A_{\gamma}(E, \Theta(t)) f(E) d E d t \quad \text { events day }{ }^{-1}
$$

is evaluated for the three spectral functions $f(E)$. In the above integral, $A_{\gamma}(E, \Theta)$ is the effective area for gamma rays with energy $E$ arriving from a zenith angle $\Theta$. The calculation of the effective area includes the effect of the

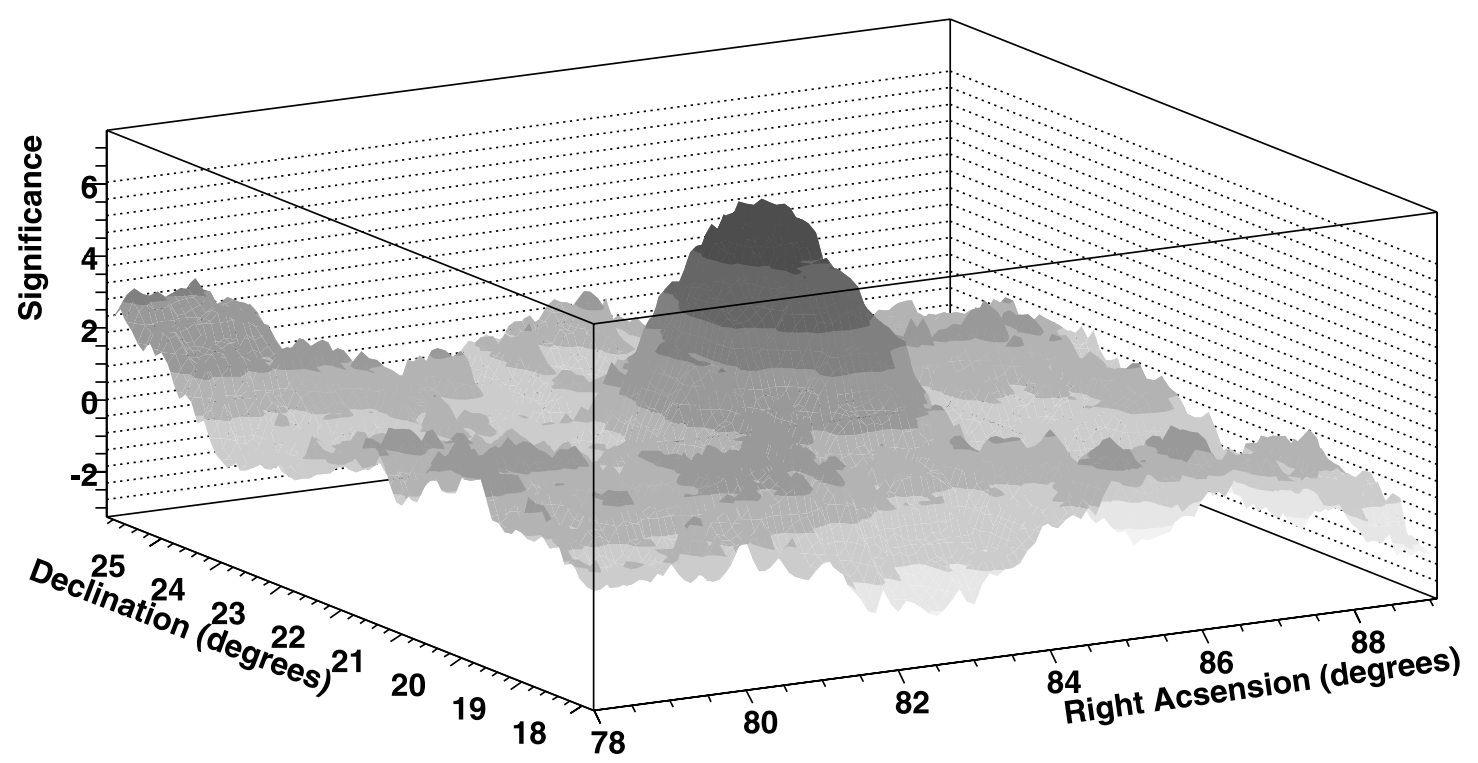

FIG. 10.-Map of the statistical significances in the region around the Crab Nebula. The $z$-axis is in standard deviations. 
TABLE 4

The Measured Flux from the Crab Nebula Assuming Four Different Spectral Shapes As Determined by Other Instruments

\begin{tabular}{|c|c|c|c|}
\hline \multirow[b]{2}{*}{ ENERGY SPECTRUM } & \multicolumn{2}{|c|}{$\begin{array}{c}I_{0} \\
\left(\times 10^{-7} \mathrm{~m}^{-2} \mathrm{~s}^{-1} \mathrm{TeV}^{-1}\right)\end{array}$} & \multirow[b]{2}{*}{ REFERENCE } \\
\hline & These Data & From Reference & \\
\hline$E^{-2.49} \ldots \ldots \ldots \ldots \ldots \ldots$ & $2.38 \pm 0.38 \pm 1.2$ & $3.20 \pm 0.17 \pm 0.6$ & 1 \\
\hline$E^{-2.44-0.151 \log _{10} E}$. & $2.71 \pm 0.44 \pm 1.4$ & $3.25 \pm 0.14 \pm 0.6$ & 1 \\
\hline$E^{-2.59}$ & $2.68 \pm 0.42 \pm 1.4$ & $2.79 \pm 0.022 \pm 0.5$ & 2 \\
\hline$E^{-2.62} \ldots \ldots \ldots \ldots-\ldots$ & $2.65 \pm 0.42 \pm 1.4$ & $8.2 \pm 1.6$ & 3 \\
\hline
\end{tabular}

Notes.-The first error is statistical and the second error is systematic. In the case of the last row, no systematic error is given in the reference.

ReFERENCES. - (1) Hillas et al. 1998; (2) Aharonian et al. 2000; (3) Amenomori et al. 1999.

background rejection criteria $(C>2.5)$, the angular reconstruction (the reconstructed direction of the event must be within 1.2 of the true direction and the number of PMTs used in the fit must be greater than 20), and the trigger requirement of the detector (number of PMTs in the top layer must be greater than 60). After determining the value of the integrand, the observed (corrected) excess of 10.7 events day ${ }^{-1}$ is used to determine $I_{0}$. The results for the four different spectral shapes are given in Table 4 . The systematic errors arise from two effects: a possible miscalibration of the detector and a systematic error in the Monte Carlo simulation of the air shower and/or the response of the detector to the air shower. The first is straightforward to estimate. The use of old calibrations to reconstruct newer data results in a $10 \%$ change in the observed signal level. Systematic errors in the simulation are more difficult to determine, and an exhaustive study has not been performed. The simulation of the air shower relies on knowledge of the particle interactions and the atmospheric model. The simulation of the response of the detector relies on knowledge of the optical properties of the water, the optical properties of the water/ cover boundary, the sensitivity of the PMTs, and the response of the electronics. With the exception of the water cover interface, these parameters have been measured, and the water quality is routinely measured. In principle, the displacement of the shadow of the Moon from its true position (due to the deflection of charged particles in the Earth's magnetic field) can be used to calibrate the energy response of Milagro. This analysis will be the subject of a future paper. Here we take a somewhat conservative value of $20 \%$ as a possible systematic error in the energy scale determined by the Monte Carlo simulations. This leads to a $\sim 60 \%$ systematic error in the determination of $I_{0}$, which dominates the systematic error of the instrument.

Table 4 indicates that while the flux measured by Milagro is in good agreement with the air Cerenkov experiments, there is a disagreement with the results from the Tibet air shower array. Including the systematic errors from Milagro, the results disagree at the $2.6 \sigma$ level; the reference for the Tibet result (Amenomori et al. 1999) does not discuss systematic errors. Given the large systematic errors, one cannot make a definitive statement, but among the other three results, the data seem to favor the softer spectra as measured by HEGRA or the curved spectrum as measured by Whipple.

\section{CONCLUSIONS}

The bottom layer of Milagro is an imaging calorimeter that can be used to measure the lateral distribution of energy deposited in Milagro. Hadronic cosmic rays generate air showers with penetrating particles that deposit localized clumps of energy in the Milagro detector. A simple algorithm to differentiate air showers induced by hadronic cosmic rays from those induced by gamma rays has been developed. This simple cut, based on a compactness parameter $\left(\mathrm{NB} / \mathrm{PEMax}_{B}\right)$, improves the sensitivity of Milagro by a factor of $\sim 1.7$. We have used this cut to observe $\mathrm{TeV}$ gamma-ray emission from the Crab Nebula. The measured flux is consistent with previous measurements by atmospheric Cerenkov telescopes, but it is inconsistent with the reported result from the Tibet air shower array.

This is the first demonstration of the ability of an EAS array to reject hadrons and enhance the significance of an observation of a source of $\mathrm{TeV}$ gamma rays. More complex techniques that utilize additional information to improve the background rejection capabilities of the instrument are under investigation. In addition, an outrigger array that is currently under construction will provide knowledge of the core location of each event, which is expected to further increase the discrimination power of Milagro. Since Milagro is a new and unique type of instrument, we are only beginning to understand its response to cosmic rays and gamma rays. As understanding of this new instrument improves, further improvements to the sensitivity of Milagro are expected.

This work has been supported by the National Science Foundation, the US Department of Energy (Office of HighEnergy Physics and Office of Nuclear Physics), Los Alamos National Laboratory, the University of California, and the Institute of Geophysics and Planetary Physics.

\section{REFERENCES}

Aharonian, F. A., et al. 2000, ApJ, 539, 317

Alexandreas, D. E., et al. 1992, Nucl. Instrum. Methods Phys. Res. A, 311, 350

1993, Nucl. Instrum. Methods Phys. Res. A, 238, 570

Amenomori, M., et al. 1999, ApJ, 525, L93
Atkins, R., et al. 2000, Nucl. Instrum. Methods Phys. Res. A, 449, 478

.2001, preprint (astro-ph/0110513)

Borione, A., et al. 1994, Nucl. Instrum. Methods Phys. Res. A, 346, 329

. 1997, Phys. Rev. D, 55, 1714

Galbraith, W., \& Jelley, J. V. 1953, Nature, 171, 349 
Hillas, A. M. 1985, Proc. 19th Int. Cosmic-Ray Conf. (La Jolla), 3, 445 Hillas, A.M., et al. 1998, ApJ, 503, 744

Hoffman, C. M., Sinnis, C., Fleury, P., \& Punch, M. 1999, Rev. Mod. Phys., 71, 897

Knapp, J., \& Heck, A. 1993, Extensive Air Shower Simulation with CORSIKA: A User's Manual (KfK 5196 B; Karlsruhe: Kernforschungszentrum Karlsruhe)
Li, T. P., \& Ma, Y. Q. 1983, ApJ, 272, 317

Nesterova, N. M., \& Chudakov, A. E. 1955, Soviet Phys.-JETP, 28, 384

Ong, R. 1998, Phys. Rep., 305, 93

Weekes, T. C., \& Turver, K. E. 1977, in Recent Advances in Gamma-Ray Astronomy: Proc. 12th ESLAB Symp., ed. R. D. Wills \& B. Battrick (ESA SP-124; Noordwijk: ESA), 279

Weekes, T. C., et al. 1989, ApJ, 342, 379 